\title{
Downregulation of miR-34a contributes to the proliferation and migration of laryngeal carcinoma cells by targeting cyclin D1
}

\author{
$\mathrm{JIN} \mathrm{YE}^{1^{*}}$, LAISHENG LI $^{2 *}$, PINNING FENG ${ }^{2}$, JIANXIN WAN $^{2}$ and JINGJIA LI \\ ${ }^{1}$ Department of Otolaryngology, Head and Neck Surgery, The Third Affiliated Hospital of Sun Yat-sen University, \\ Guangzhou, Guangdong 510630; ${ }^{2}$ Department of Laboratory Medicine, The First Affiliated Hospital \\ of Sun Yat-sen University, Guangzhou, Guangdong 510080, P.R. China
}

Received December 29, 2015; Accepted February 11, 2016

DOI: $10.3892 /$ or.2016.4823

\begin{abstract}
Laryngeal carcinoma is one of the most common head and neck cancers. MicroRNAs are a class of small non-coding RNAs 18-25 nucleotides in length that posttranscriptionally regulate gene expression and have a pivotal role in many biological processes including cancer development. In this study, we investigated the role of miR-34a in laryngeal carcinoma and confirmed the regulation of cyclin D1 (CCND1) by miR-34a. We examined miR-34a expression levels in 71 laryngeal carcinoma patient specimens by quantitative reverse transcription-PCR, and analyzed the clinicopathological significance of the obtained data. Then, functional assays were performed to investigate the potential effects of miR-34a on cancer cell proliferation and migration. In addition, western blotting, luciferase reporter assay and several algorithms were conducted to confirm that CCND1 is directly regulated by miR-34a. We demonstrated that the miR-34a expression level was significantly downregulated in laryngeal carcinoma clinical specimens compared with that observed in their paired adjacent normal tissues. Additionally, miR-34a expression was also inversely correlated with lymph node metastasis and clinical stage. Functional assays showed that ectopic expression of miR-34a inhibited cell proliferation and migration in laryngeal carcinoma cells. Bioinformatic analysis identified CCND1 as a potential target of miR-34a.
\end{abstract}

Correspondence to: Dr Jin Ye, Department of Otolaryngology, Head and Neck Surgery, The Third Affiliated Hospital of Sun Yat-sen University, 600 Tianhe Road, Tianhe, Guangzhou 510630, P.R. China

E-mail: yejin_sums@aliyun.com

Dr Laisheng Li, Department of Laboratory Medicine, The First Affiliated Hospital of Sun Yat-sen University, 58 Zhongshan Road, Guangzhou 510080, P.R. China

E-mail: lilaish@mail.sysu.edu.cn

*Contributed equally

Key words: miR-34a, laryngeal carcinoma, cyclin D1, proliferation, tumor suppressor
Moreover, we confirmed that miR-34a inhibited the expression of CCND1 by directly binding to its 3'-untranslated region. Silencing of CCND1 induced effects similar to those of miR-34a ectopic expression, and in laryngeal carcinoma tissues, miR-34a and CCND1 were inversely correlated. Our data suggest that tumor suppressor miR-34a could serve as a new potential diagnostic marker and that ectopic expression of miR-34a may be used as a therapeutic target for laryngeal carcinoma.

\section{Introduction}

Laryngeal carcinoma is one of the most commonly diagnosed head and neck cancers worldwide. Owing to advances in both diagnostic techniques and comprehensive treatment, the therapeutic efficacy is good in early stage disease. However, the survival rate of advanced stage laryngeal carcinoma patients has not significantly improved over recent years (1). Once recurrence or distant metastasis presents, conventional therapy is almost ineffective. Therefore, research has focused on the development of novel molecular mechanisms underlying laryngeal carcinoma progression and on the exploration of effective schemes for diagnosis and therapy of this disease.

MicroRNAs (miRNAs) are endogenous non-coding small RNAs, roughly 18-25 nucleotides in length that contribute to the translation inhibition or degradation of their cognate target genes through an imperfect sequence complementarity-dependent manner by which to pair the 3'-untranslated region (3'-UTR) of a target mRNA (2). Studies have demonstrated that each miRNA may regulate hundreds of target genes and half of miRNAs are located in cancer-associated genomic regions (3). In fact, miRNAs are involved in entire signaling networks to regulate several cellular processes, including proliferation, differentiation and apoptosis (4). Evidence has shown that a number of miRNAs are involved in tumorigenesis and function either as oncogenes or tumor suppressors during tumor progression $(5,6)$. Therefore, aberrant expression profiles of miRNAs have considerable potential for the diagnosis, classification, clinical prognostic information, and therapy of cancer (7-9).

Previous studies of miRNA profiles have confirmed miR-34a as a key regulator of tumor suppression, and miR-34a is downregulated in many solid and hematological malignan- 
cies, including laryngeal carcinoma (10-12). miR-34a is an important component of the p53 tumor suppressor network, whose gene resides on chromosome 1p36.23, and is always deregulated by gene deletion, $\mathrm{CpG}$ methylation or mutation of p53 (13). Ectopic expression of miR-34a can induce apoptosis, cell cycle arrest, senescence, differentiation, and self-renewal, by regulating several target mRNAs, such as c-Myc, E2F, CDK4, CDK6, Bcl-2, SIRT1, c-MET and CD44, to inhibit cancer proliferation and metastasis in various types of cancers (14-21). Our study showed that miR-34a was downregulated in laryngeal carcinoma tissues, and a bioinformatic analysis predicted CCND1 as a potential target of miR-34a.

CCND1 is an established human oncogene that is frequently overexpressed as a result of copy number variation, mutation, or as a consequence of the deregulation of miRNAs (22). The fundamental function of CCND1 is to promote tumor progression through activation of the cyclin-dependent kinases (CDKs) CDK4 and CDK6 and inactivation of the retinoblastoma protein and release of E2F $(23,24)$. Various human cancers, including breast cancer, colon cancer, lung cancer, melanoma, prostate cancer, hematopoietic malignancies and laryngeal carcinoma, overexpress CCND1 (25). In laryngeal carcinoma, overexpression of CCND1 is associated with unfavorable clinicopathological features and represents an independent significant predictor of patient prognosis (26). miR-34a was found to induce cell cycle arrest through regulation of CCND1 in non-small cell lung cancer $(17,27)$. However, the potential mechanisms by which CCND1 affects the malignant phenotype of laryngeal carcinoma cells remain largely unknown.

In this study, we investigated the role of miR-34a in the development and progression of laryngeal carcinoma. We examined the expression levels of miR-34a in laryngeal carcinoma cells and clinical samples and tested its effects on cell proliferation, apoptosis and migration. In addition, we identified that CCND1 was overexpressed in laryngeal carcinoma and predicted that CCND1 was a target of miR-34a, by 3'-UTR luciferase assays and western blot analysis. Furthermore, we validated CCND1 as the main functional target of miR-34a through knockdown and rescue expression of CCND1 in laryngeal carcinoma. Our study demonstrated that miR-34a acts as a tumor suppressor by the direct targeting of CCND1 in laryngeal carcinoma, suggesting that miR-34a is a potential diagnostic biomarker and has therapeutic value for laryngeal carcinoma therapy.

\section{Materials and methods}

Patient tissues. Seventy-one cases of human laryngeal squamous cell carcinoma (LSCC) tissue specimens (tumor tissues with corresponding normal adjacent tissues) were obtained at the time of surgical resection from the Third Affiliated Hospital of Sun Yat-sen University (Guangzhou, Guangdong, China) between January 2011 and September 2014. Written informed consent was obtained from all patients for the use of their specimens before surgery. The specimens were conserved in RNAlater (Ambion, Austin, TX, USA) immediately after surgical resection and transferred into $-80^{\circ} \mathrm{C}$ freezer until use. The histological characterization and clinicopathological staging of the LSCC tissues were determined according to the current International Union Against Cancer (UICC) pathological staging criteria. Use of the human tissues was approved by the Clinical Research Ethics Committee of the Third Affiliated Hospital of Sun Yat-sen University.

Cell culture. The human laryngeal carcinoma cell line HEp-2 was obtained from the American Type Culture Collection (ATCC, Manassas, VA, USA) and was freshly recovered from liquid nitrogen (less than 3 months). HEp-2 cells were maintained in RPMI-1640 medium (Invitrogen, Beijing, China). Media were supplemented with $10 \%$ fetal bovine serum (FBS; Gibco, Cappinas, Brazil). All cells were cultured in a humidified incubator (Thermo Electron Corp., New Castle, DE, USA) at $37^{\circ} \mathrm{C}$ with $5 \% \mathrm{CO}_{2}$.

Bioinformatics. The potential target gene of miR-34a and its conserved miRNA-binding site was analyzed by the algorithms of TargetScan 5.1 (http://www.targetscan.org) and miRanda (http://www.microrna.org). According to these algorithms, we selected the CCDN1 gene as a potential target of miR-34a for further study.

Transient transfection of miRNA and siRNA. The miR-34a mimics, miR-Ctrl (miRNA negative control with non-specific), small interfering RNA duplexes targeting human CCND1 (CCND1-siRNA, sense strand, 5'-GGAGAACAAACAGAUC AUCTTdTdT-3' and antisense strand, 5'-GAUGAUCUGUUU GUUCUCCTCdTdT-3') and scrambled control siRNA (Ctrl-siRNA) (sense strand, 5'-UUCUCCGAACGUGUCAC GUdTdT-3' and antisense strand, 5'-ACGUGACACGUUCG GAGAAdTdT-3') were purchased from RiboBio, Co., Ltd. (Guangzhou, China). Cells were transfected at $\sim 60-70 \%$ confluence using Lipofectamine 2000 reagent (Invitrogen Life Technologies, Carlsbad, CA, USA) with miRNA mimics or siRNA duplexes at working concentrations of $50 \mathrm{nM}$ in Opti-MEM (Invitrogen Life Technologies) according to the protocol as previously described (w).

RNA extraction, reverse transcription and quantitative real-time PCR ( $q P C R)$. The total RNA extraction, reverse transcription and $\mathrm{qPCR}$ procedure were executed as previously described (20). In brief, total RNA from the cultured cells and surgical LSCC tissues were extracted by TRIzol ${ }^{\circledR}$ reagent (Invitrogen Life Technologies). To quantitate the expression level of miR-34a, reverse transcription was carried out by a specific stem-loop real-time PCR miRNA kit (RiboBio, Co., Ltd.). After reverse transcription at $42^{\circ} \mathrm{C}$ for $60 \mathrm{~min}$ followed by denaturation at $70^{\circ} \mathrm{C}$ for $10 \mathrm{~min}, \mathrm{qPCR}$ was carried out using the Platinum SYBR Green qPCR SuperMix-UDG system (Invitrogen Life Technologies) on an ABI 7900HT Real-Time PCR system (Applied Biosystems Life Technologies, Foster City, CA, USA). Data were analyzed by ABI SDS version 2.3 (Applied Biosystems Life Technologies). All procedures were performed according to the protocols of the manufacturer. Each sample was analyzed in triplicate and normalized to internal controls, 5S ribosomal RNA, and the fold changes were calculated according to the relative quantification method ( $R Q=$ $\left.2^{-\Delta \Delta \mathrm{CT}}\right)$. The results are expressed as fold-change in the expression levels in the cells or tissues. 
The primers of miR-34a and 5S rRNA used for stem-loop real-time PCR are listed as follows: miR-34a stem-loop RT, 5'-GTCGTATCCAGTGCAGGGTCCGAGGTATTCGCACT GGATACGAAGGGCAG-3'; miR-34a forward, 5'-GCGGC CAATCAGCAAGTATACT-3'; miR-34a reverse, 5'-GTGCA GGTCCGAGGT-3'; 5S rRNA stem-loop RT, 5'-GTCGTATC CAGTGCAGGGTCCGAGGTATCGCACTGGATACGACC AGGCG-3'; 5S rRNA forward, 5'-CTGGTTAGTACTTGG ACGGGAGAC-3'; 5S rRNA reverse, 5'-GTGCAGGGTCCG AGGT-3'.

MTT assay. The cell growth and proliferation of the HEp-2 cells transfected with miRNAs or siRNAs were assessed by 3-(4,5-dimethylthiazol-2-yl)-2,5-diphenyltetrazolium bromide (MTT) assay (Sigma-Aldrich, St. Louis, MO, USA). The cells were plated in 96-well plates at $5 \times 10^{3}$ cells/well and transfected with miRNAs or siRNAs. After incubation at the appropriate time points, the culture medium was replaced by $100 \mu \mathrm{l}$ of fresh DMEM. And $25 \mu \mathrm{l}$ of MTT $(5 \mathrm{~g} / \mathrm{l}$ in phosphatebuffered saline) was added to each well, to obtain a working concentration of MTT, $1 \mathrm{~g} / 1$. Then, incubation was carried out for an additional $4 \mathrm{~h}$, the medium was replaced with dimethyl sulfoxide (DMSO; Sigma-Aldrich), and the absorbance was determined using a SpectraMax M5 microplate reader (Molecular Devices, LLC, Sunnyvale, CA, USA) at $570 \mathrm{~nm}$. The cell viability was normalized to the cells without transfection of the miRNA or siRNA. Three independent experiments (triplicate in each) were analyzed.

Apoptosis assays. To determine cell apoptosis in vitro, we used the Annexin V-fluorescein isothiocyanate apoptosis detection kit (KeyGen, Nanjing, China) according to the manufacturer's instructions. Briefly, after a 48-h transfection, the cells were collected by trypsinization and washed with PBS, and resuspended in binding buffer at a concentration of $1 \times 10^{6}$ cells $/ \mathrm{ml}$. Next, the cells were incubated with $2 \mu 1$ of propidium iodide and $2 \mu \mathrm{l}$ of Annexin V-fluorescein isothiocyanate for $15 \mathrm{~min}$, washed and resuspended in $500 \mu 1$ PBS. Flow cytometric analysis was carried out by a FACSCalibur (Becton-Dickinson, San Jose, CA, USA).

Transwell invasion assay. To evaluate cell migration in vitro, Transwell chambers ( $8 \mu \mathrm{m}$; BD Biosciences, San Jose, CA, USA) were used according to the manufacturer's instructions. Briefly, the transfected cells were collected, re-suspended $\left(5 \times 10^{4}\right.$ cells/well) in $200 \mu 1$ serum-free medium, and added to the top chamber. In the bottom chamber, culture medium containing $10 \%$ FBS served as a chemoattractant. After incubation for $24 \mathrm{~h}$ at $37^{\circ} \mathrm{C}$, the cells that did not migration through the pores were carefully removed by cotton swabs. Then the invasive cells were fixed and stained with $0.5 \%$ crystal violet (Sigma-Aldrich) for $30 \mathrm{~min}$, counted with an inverted microscope (Olympus IX71; Olympus Corp., Tokyo, Japan), and the relative number of migratory cells was calculated from five random fields from digital images (x200). The data are expressed as the means \pm SD of three independent experiments.

Plasmid. The 3'-UTR sequences of human CCND1 containing the putative miR-34a binding sites were obtained from healthy and disease-free volunteer gDNA using PCR amplification and cloned into the pGL3 luciferase reporter plasmid (Promega, Corp., Madison, WI, USA), which was termed as wild-type 3'-UTR (wt 3'-UTR). The point mutations were created by the Quick-Change Site-Directed Mutagenesis kit (Stratagene, La Jolla, CA, USA), according to the manufacturer's instructions. The resultant product served as the mutated 3'-UTR (mut 3'-UTR). Both inserted fragment sequences were confirmed by DNA sequencing.

To overexpress CCND1, the cDNA of CCND1 containing the putative miR-34a binding sites was cloned into the pcDNA3.1 vector (Invitrogen Life Technologies), which was termed as wild-type 3'-UTR-CCND1 (wt 3'-UTR-CCND1). The mut 3'-UTR-CCND1 was obtained as described above. Cells were co-transfected with $50 \mathrm{nM}$ of miRNA mimics and $500 \mathrm{ng}$ of plasmid in a 6-well plate for the rescue experiment.

Luciferase assays. For the luciferase reporter assay, HEp-2 cells $\left(6 \times 10^{4}\right)$ were seeded in triplicate in 24-well plates $24 \mathrm{~h}$ before transfection. Vectors, as described above, and the control vector pRL-TK (Promega, Corp.) coding for Renilla luciferase (for normalization) were co-transfected with miRNA in triplicate by Lipofectamine 2000. The cells were harvested and lysed $48 \mathrm{~h}$ after transfection, and the luciferase activity was measured using the Dual-Glo luciferase assay kit (Promega, Corp.). The firefly luciferase values were normalized to Renilla, and the relative ratios of firefly to Renilla activity are shown. Three independent experiments were carried out, and the data are represented as the mean \pm SD.

Western blot analysis. According to standard western blot analysis procedures, briefly, after culturing for $72 \mathrm{~h}$, the transfected cells were harvested on ice using RIPA lysis and extraction buffer [ $25 \mathrm{mM}$ Tris- $\mathrm{HCl} \mathrm{pH} 7.6,150 \mathrm{mM} \mathrm{NaCl}$, $1 \%$ NP-40, $1 \%$ sodium deoxycholate, $0.1 \%$ SDS, protease inhibitor cocktail (Pierce, Rockford, IL, USA)]. Then, $10 \mu \mathrm{g}$ protein of each sample was separated by sodium dodecyl sulfate polyacrylamide gel electrophoresis (SDS-PAGE) on 10\% polyacrylamide gels, and then transferred to polyvinlidene fluoride membranes (PVDF; Millipore, Billerica, MA, USA). After blocking for $1 \mathrm{~h}$ at room temperature and incubation overnight at $4^{\circ} \mathrm{C}$ in Tris-buffered saline containing $0.05 \%$ Tween (TBST) with $5 \%$ milk, the membranes were incubated with mouse monoclonal antibody against human CCND1 (Cell Signaling Technology, Danvers, MA, USA) or tubulin (Sigma-Aldrich) as a protein loading control, followed by horseradish peroxidase (HRP)-conjugated goat-anti-mouse IgG (Abcam), and the bands were detected using the Supersignal West Pico ECL chemiluminescence kit (Pierce) and Kodak X-ray film (Eastman Kodak Co, Rochester, NY, USA).

Statistical analysis. SPSS 13.0 software was used for statistical analysis. All experiments were performed independently three times, and all samples were in triplicate. The data are expressed as the mean \pm SEM unless otherwise mentioned; two-tailed Student's t-test was used for statistical analysis when only two groups were tested. A one-way analysis of variance was used to compare multiple groups. Spearman's correlation analysis was used to determine the correlation between miR-34a and CCND1 expression. P-values of $<0.05$ were considered to indicate statistical significance. 
Table I. Relationship between miR-34a expression and clinicopathological parameters of the laryngeal cancer cases.

\begin{tabular}{|c|c|c|c|}
\hline $\begin{array}{l}\text { Clinicopathological } \\
\text { parameters }\end{array}$ & $\begin{array}{l}\text { No. of } \\
\text { cases }\end{array}$ & $\begin{array}{l}\text { Median expression } \\
\text { of miR-34a }\end{array}$ & P-value \\
\hline \multicolumn{4}{|l|}{ Gender } \\
\hline Male & 56 & $0.3432 \pm 0.0533$ & 0.6160 \\
\hline Female & 15 & $0.3487 \pm 0.0616$ & \\
\hline \multicolumn{4}{|l|}{ Age (years) } \\
\hline$\leq 60$ & 33 & $0.3854 \pm 0.0631$ & 0.5328 \\
\hline$>60$ & 38 & $0.3225 \pm 0.0535$ & \\
\hline \multicolumn{4}{|l|}{ Primary location } \\
\hline Supraglottic & 29 & $0.3024 \pm 0.0567$ & 0.2869 \\
\hline Glottic & 42 & $0.3579 \pm 0.0566$ & \\
\hline \multicolumn{4}{|c|}{ Lymph node metastasis } \\
\hline Negative & 43 & $0.5028 \pm 0.0551$ & 0.0006 \\
\hline Positive & 28 & $0.2202 \pm 0.0441$ & \\
\hline \multicolumn{4}{|l|}{ Differentiation } \\
\hline Well & 45 & $0.3990 \pm 0.0562$ & 0.0079 \\
\hline Moderate and low & 26 & $0.2317 \pm 0.0430$ & \\
\hline \multicolumn{4}{|l|}{$\mathrm{T}$ classification } \\
\hline $\mathrm{T} 1+\mathrm{T} 2$ & 44 & $0.4029 \pm 0.0576$ & 0.0380 \\
\hline $\mathrm{T} 3+\mathrm{T} 4$ & 27 & $0.2641 \pm 0.0460$ & \\
\hline \multicolumn{4}{|l|}{ Clinical stage } \\
\hline $\mathrm{I}+\mathrm{II}$ & 39 & $0.5250 \pm 0.0578$ & 0.0001 \\
\hline III+IV & 32 & $0.2202 \pm 0.0392$ & \\
\hline
\end{tabular}

\section{Results and Discussion}

miR-34a is downregulated in human LSCC specimens and is inversely associated with advanced stage and lymph node metastasis in the patients. To investigate the expression of miR-34a and its significance in LSCC, we first examined the expression levels of miR-34a in 71 pairs of primary LSCC and their corresponding normal adjacent tissues by stem-loop RT-PCR. The correlation between the miR-34a expression levels and the clinicopathological characteristics of the LSCC patients, including gender, age, primary location, lymph node status, $T$ classification and clinical stage are summarized in Table I. The data demonstrated no obvious correlations between miR-34a expression and gender, age and primary location. However, as shown in Fig. 1A, the comparative analysis indicated that the median expression level of miR-34a was lower in the LSCC tissues compared with the median level in the paired normal adjacent tissues (median, 0.3432 and 0.9756 , respectively; $\mathrm{P}<0.0001$ ). In regards to lymph node status, we separated 71 LSCC patients into two groups: lymphatic node metastasis-positive or -negative. Notably, miR-34a expression was significantly lower in the lymphatic node metastasis-positive group than that in the negative group (Table I and Fig. 1B; P=0.0006). Furthermore, the expression of miR-34a was lower in the LSCC cases with advanced TNM stage (stage III and IV), compared to that in early stage LSCC patients (stage I and II; Table I and Fig. 1C). Collectively, these data suggested that miR-34a was downregulated in LSCC and
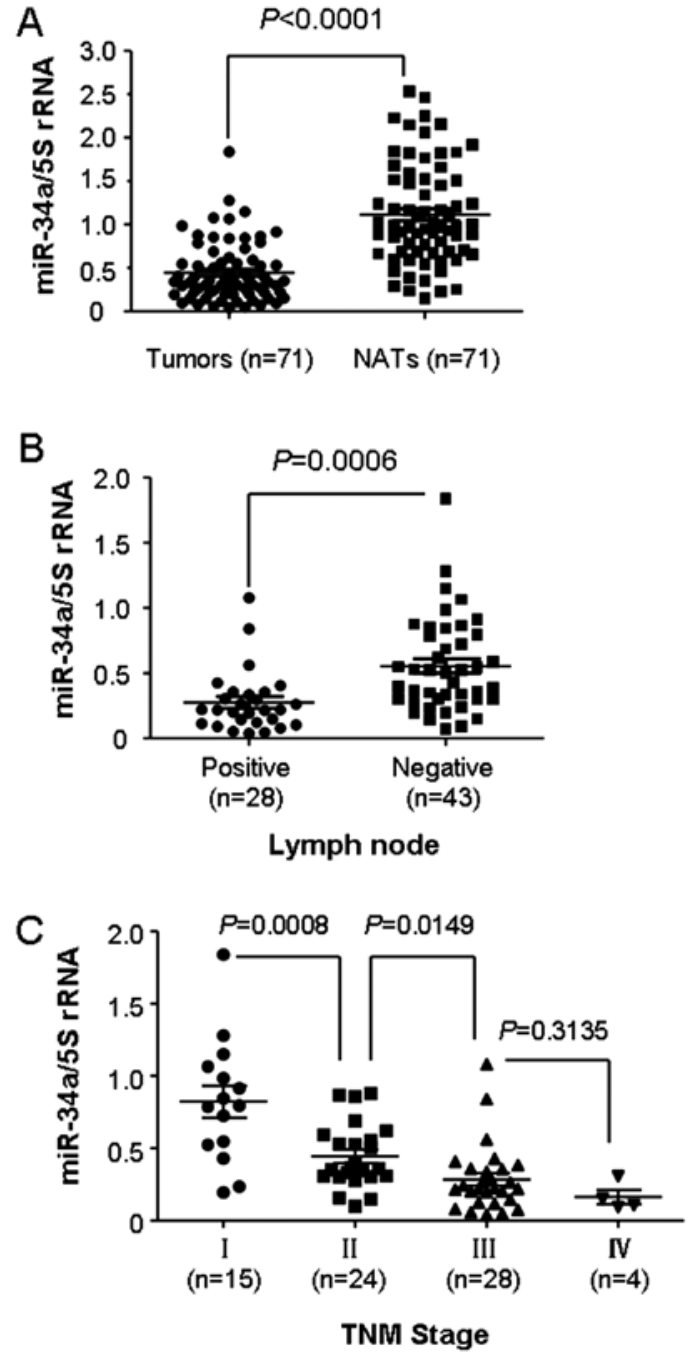

Figure 1. Expression levels of miR-34a in human LSCC specimens. (A) Expression of miR-34a in 71 cases of LSCC and their paired normal adjacent tissues; miR-34a expression was determined by qRT-PCR and normalized to 5S rRNA. (B) The expression level of miR-34a is associated with lymph node metastasis of LSCC $(\mathrm{P}=0.0006)$. (C) The expression levels of miR-34a in LSCC patients with different clinical stages. Statistical analysis was performed using the paired t-test (A) and the Student's t-test (B and C). $\mathrm{y}$-axis indicates relative miR-34a expression levels which were calculated as the ratio of miR-34a to the internal control, $5 \mathrm{~S}$ rRNA, according to the relative quantification equation $R Q=2^{-\Delta \Delta C T}$. Data are expressed as the mean \pm SD from three independent experiments. ${ }^{*} \mathrm{P}<0.05$. NATs, normal adjacent tissues; LSCC, laryngeal squamous cell carcinomas.

a decreased expression of miR-34a could be attributed to the progression and metastasis of LSCC.

miR-34a inhibits the proliferation, migration and induces apoptosis in the HEp-2 cells. To explore the function of miR-34a on LSCC cell proliferation, we transfected LSCC HEp-2 cells with miR-34a mimics. The effectiveness of miR-34a ectopic overexpression in the HEp-2 cells was tested by quantitative real-time PCR. Compared with the negative control (miR-Ctrl), the mimics significantly elevated the expression of miR-34a (Fig. 2A). As shown in the MTT assay, restoration of miR-34a inhibited the proliferation of HEp-2 cells, compared with the miR-Ctrl (Fig. 2B). Then, we observed the morphologic images of the HEp-2 cells transfected with the 
A

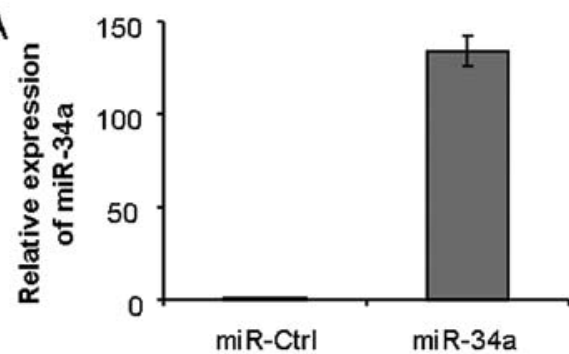

C

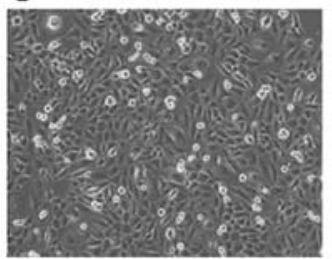

miR-Ctrl

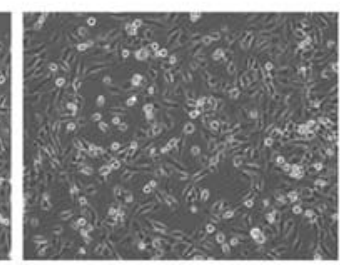

miR-34a
B

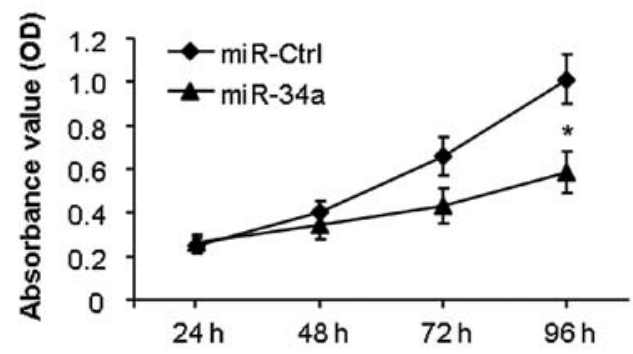

D

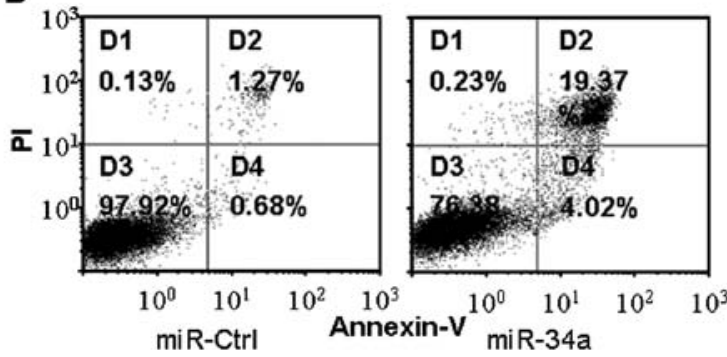

E

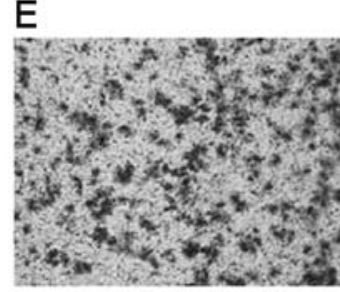

miR-Ctrl

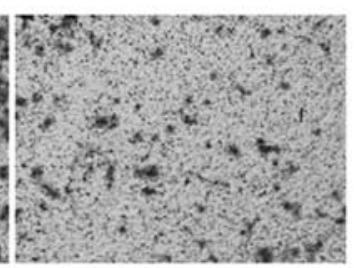

miR-34a

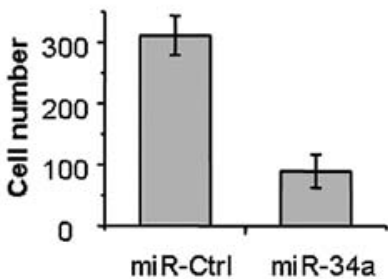

Figure 2. miR-34a suppresses HEp-2 cell proliferation, migration and induces apoptosis. (A) miR-34a was re-expressed in HEp-2 cells by transfection with miR-34a mimics. Forty-eight hours later, the miR-34a expression levels were measured by quantitative RT-PCR and normalized to 5S rRNA. (B) Effect of miR-34a on cell proliferation was tested by MTT assay. (C) Morphologic changes in HEp-2 cells in response to miR-34a expression. (D) Apoptosis analysis of HEp-2 cells in response to miR-34a at 48 h, by FACS assay. (E) Analysis of the effect of miR-34a on the migration of HEp-2 cells by Transwell migration assay. The data represent the mean values of three independent experiments. ${ }^{*} \mathrm{P}<0.05$, with paired $t$-test.

A

miR-34a 3'
Human 5'
Rhesus 5'
Mouse 5'
Rat 5'
Dog 5'
Horse 5'
Cat 5'

Seed region

...GGUCGAUUCUGUGACGGU

ACAAUGUCAUAUACUGCOAUGUACU 3'

ACAAUGUCAUAUACUGCOAUGUACU 3'

ACAAUGUCAUAUACUGCOAUGUACU 3' ACAAUGUC- - AGACUGCOAUGUACU 3' ACAAUGUCAUAUACUGCOAUGUACU 3' ACAAUGUCAUAUACUGCOAUGUACU 3' ACAAUGUCAUAUACUGCOAUGUACU 3' ACGAUGUCAUAUACUGCOAUGUACU 3'
B

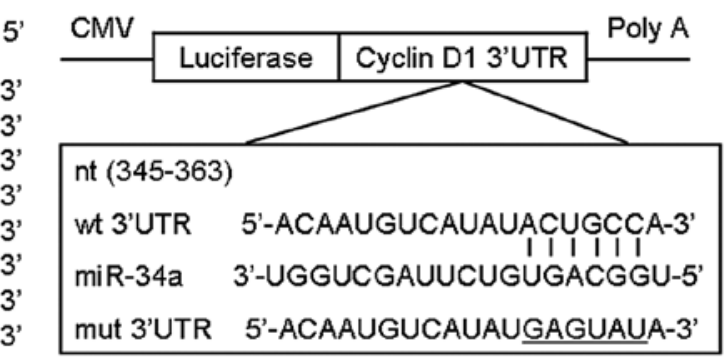

3'UTR of Cyclin D1 mRNAs
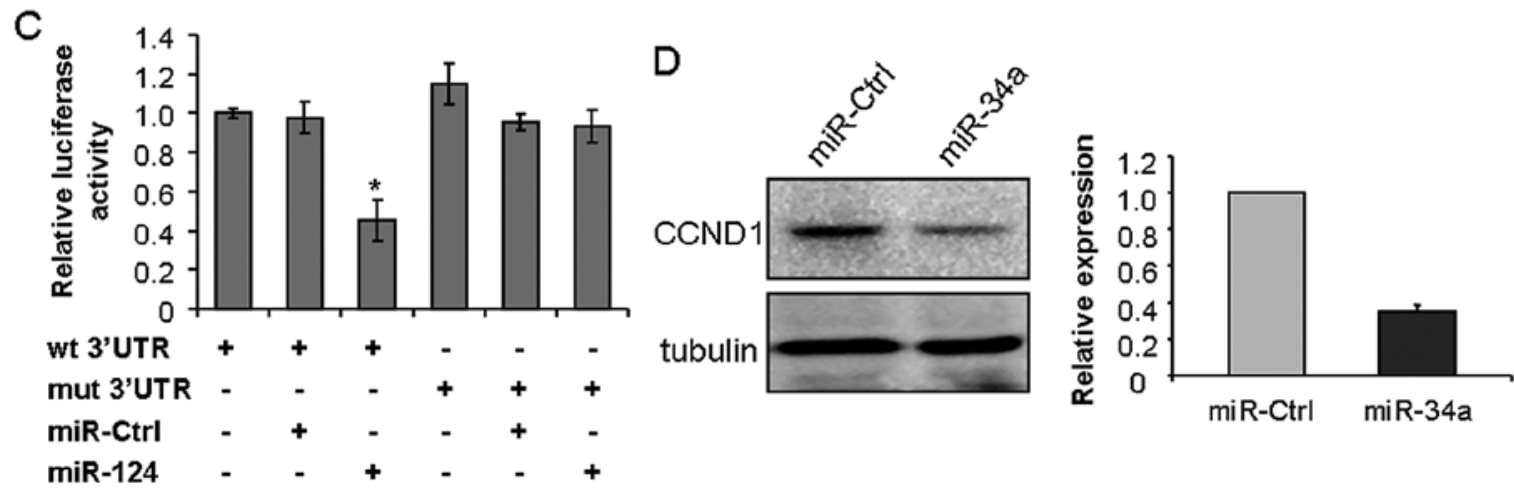

Figure 3. CCND1 is a potential target of miR-34a. (A) Schematic of the putative miR-34a binding site in the 3'-UTR region of CCND1 and interspecies conservation of seed matching sequences (gray box). (B) Diagram of CCND1 3'-UTR containing the reporter constructs. (C) Luciferase reporter assays in HEp-2 cells co-transfected with wt/mut 3'-UTR and miR-34a/miR-Ctrl as indicated. (D) Expression levels of CCND1 were tested after miR-34a transfection at $50 \mathrm{nM}$ in HEp- 2 cells by western blotting assay. ${ }^{*} \mathrm{P}<0.05$ compared with the control. 3'-UTR, 3'-untranslated region. 

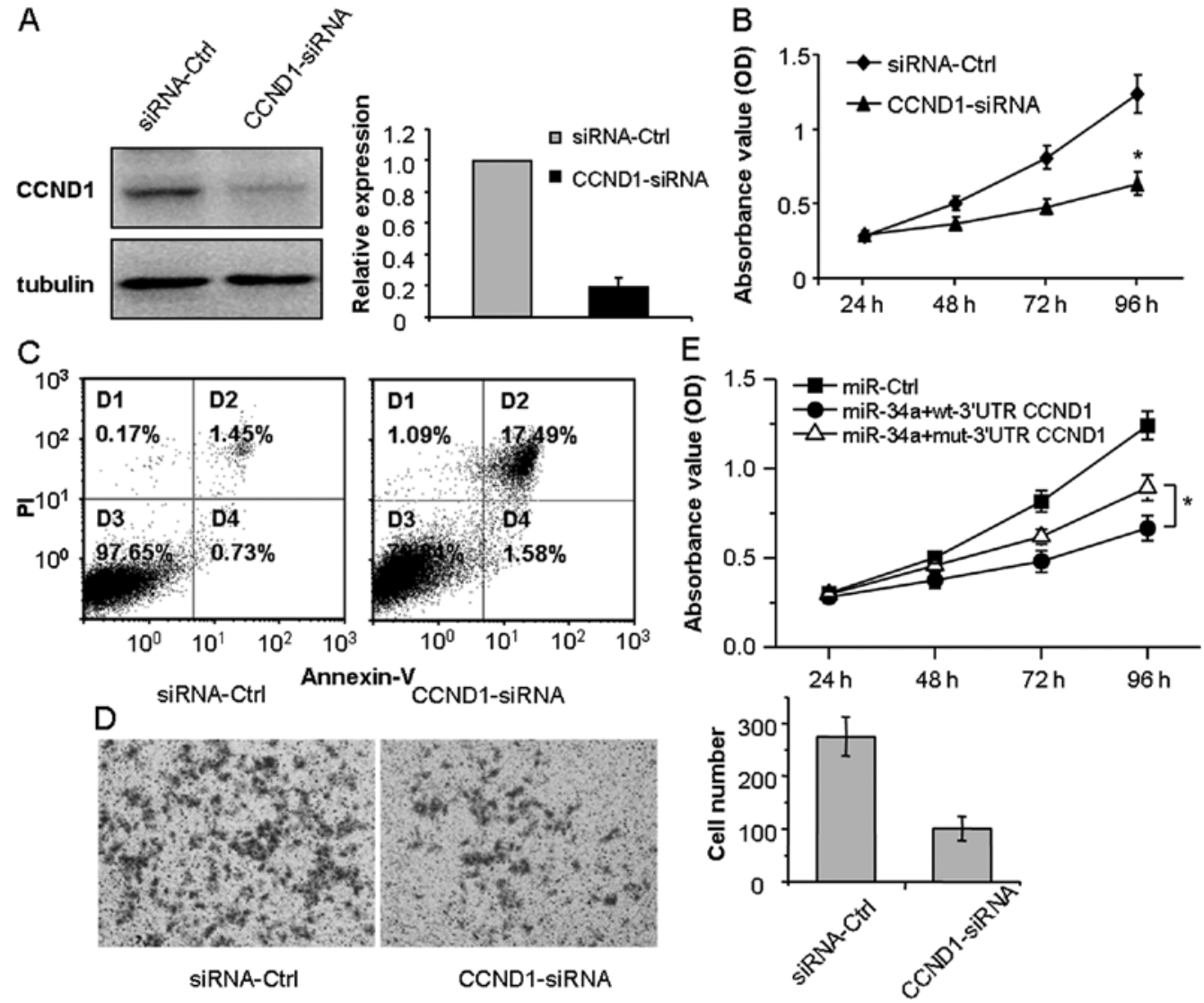

Figure 4. CCND1 is essential for LSCC proliferation and migration, and is involved in the miR-34a-induced effect. (A) Analysis of CCND1 protein expression by western blot analysis. CCND1 protein was reduced by small interfering RNA (CCND1-siRNA) in the HEp-2 cells. (B) MTT assay showed that cell proliferation was inhibited in the HEp-2 cells after transfection with CCND1-siRNA, compared with the scrambled sequence (Ctrl-siRNA). (C) Apoptosis analysis of HEp-2 cells in response to CCND1-siRNA at $48 \mathrm{~h}$, by FACS assay. (D) Analysis of the effect of CCND1-siRNA on the migration of HEp-2 cells by Transwell migration assay. (E) MTT assay shows the cell proliferation in HEp-2 cells co-transfected with miR-34a and wt/mut 3'-UTR-CCND1 compared with miR-Ctrl. " $\mathrm{P}<0.05$ compared with the control. LSCC, laryngeal squamous cell carcinomas.
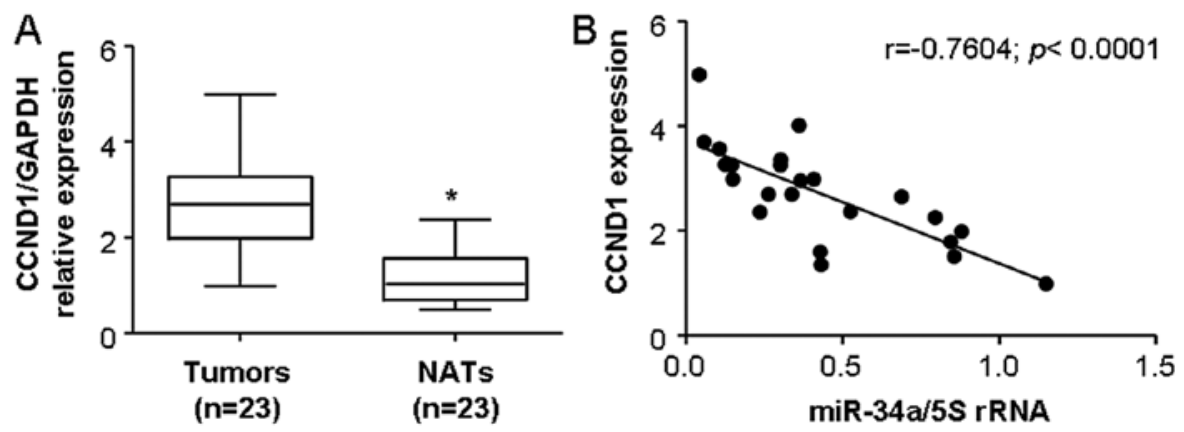

Figure 5. miR-34a and CCND1 are inversely correlated in LSCC tissues. (A) Statistical quantification of the mRNA expression levels of CCND1 (analyzed with $\mathrm{q}-\mathrm{PCR}$ ) between LSCC and paired NAT specimen. The CCND1 level was normalized to GAPDH $(\mathrm{P}<0.01)$. (B) A scatter diagram shows an inverse correlation between miR-34a and CCND1 expression in the same set of LSCC tissues (Spearman's correlation analysis, $r=-7604$; P $<0.0001$ ). NATs, normal adjacent tissues; LSCC, laryngeal squamous cell carcinomas.

miR-34a mimics. The cells exhibited significantly reduced cell numbers, compared with the miR-Ctrl-transfected cells (100 vs. $\sim 40 \%$ confluence, respectively). These data were concordant with the MTT assay. Meanwhile, the cells treated with miR-34a displayed morphological changes characterized by extension of the cytoplasmic portion and rounding of the cell body, while the rest of the spindled cells showed conspicuous shrinkage and extensive detachment (Fig. 2C).
It is well known that miR-34a is a key regulator of tumor suppression, which can induce cancer cell apoptosis. In order to explore the biological effect of miR-34a in HEp-2 cells, flow cytometric analysis of Annexin V-FITC/PI staining was used to detected apoptotics cells. As shown in Fig. 2D, compared with miR-Ctrl, transfection with miR-34a mimics obviously increased the number of apoptotic cells (1.95 vs. 23.39\%; $\mathrm{P}<0.05)$. Therefore, ectopic expression of miR-34a 
may reduce cell growth of LSCC by inhibiting cell proliferation and inducing cell apoptosis. In addition, we investigated the role of miR-34a in cell migration using Transwell assay. As shown in Fig. 2E, ectopic expression of miR-34a obviously reduced the cell migration, compared with the miR-Ctrl. Taken together, miR-34a exhibited a tumor suppressor function, and inhibited LSCC cell proliferation and migration.

miR-34a downregulates CCND1 expression by directly targeting its 3'-UTR. To explore the molecular mechanism underlying the ability of miR-34a to inhibit cancer cell proliferation and migration, bioinformatic analysis was used to identify the putative protein-coding gene targets of miR-34a, especially for those oncogenes which involved in proliferation, migration and potential therapeutic targets of cancer. According to this principle, CCND1 was chosen as one of the potential targets of miR-34a for further experimental validation, which was strictly consistent among different species and whose 3'-UTR of mRNA has one potential complementary site for the seed region of miR-34a (Fig. 3A). To test whether CCND1 is a direct target of miR-34a, the target fragment sequence of CCND1 3'-UTR (wt 3'-UTR) and the corresponding mutant counterpart sequence (mut 3'-UTR) were cloned into a luciferase reporter vector (Fig. 3B). The constructed reporter vectors as described were co-transfected with miR-34a mimics or miR-Ctrl into the HEp-2 cells. As expected, miR-34a reduced the luciferase activity of the CCND1 wt 3'-UTR by $\sim 50 \%$, while the luciferase activity was not obviously changed in the 3'-UTR with mutant binding site construct. Meanwhile, miR-Ctrl did not attenuate the luciferase activity of either the wt or mut 3'-UTR construct (Fig. 3C). In addition, western blotting results showed that ectopic expression of miR-34a significantly reduced CCND1 expression to $\sim 40 \%$ in the HEp-2 cells (Fig. 3D). These data indicated that miR-34a suppressed CCND1 expression dependent on the specific seed binding sites of the CCND1 3'-UTR.

CCNDI is involved in the effects of miR-34a on proliferation and migration. To evaluate the role of CCND1 in LSCC, HEp-2 cells were transfected with CCND1-specific siRNAs (CCND1-siRNA) or negative control (siRNA-Ctrl). After $48 \mathrm{~h}$, proteins were collected and western blotting assay showed that CCND1 protein in the cells transfected with $50 \mathrm{nM}$ CCND1-siRNA was significantly reduced (Fig. 4A). The MTT assays demonstrated that the knockdown of CCND1 suppressed the proliferation of HEp-2 cells (Fig. 4B; P<0.05). Furthermore, flow cytometric analysis indicated that the knockdown of CCND1 induced the apoptosis of HEp-2 cells (Fig. 4C; $\mathrm{P}<0.05)$. In addition, Transwell assays revealed that knockdown of CCND1 inhibited the cell migration of HEp-2 cells (Fig. 4D; $\mathrm{P}<0.05$ ). Take together, the effects of the knockdown of CCND1 in HEp-2 cells had effects similar to those of miR-34a.

To determine whether or not CCND1 is the direct functional regulator of the miR-34a effect in HEp-2 cells, we co-transfected miR-34a mimics accompanied by $\mathrm{wt} /$ mut 3'-UTR-CCND1 plasmid which contained wild-type or mutant 3'-UTR-CCND1 cDNA into HEp-2 cells. MTT results indicated that mut 3'-UTR-CCND1 slightly attenuated the miR-34a-mediated effects in the HEp-2 cells, to regain the proliferation, compared with the miR-Ctrl (Fig. 4E; $\mathrm{P}<0.05$ ).
These results suggest that CCND1 plays an important role in the regulation of miR-34a in HEp-2 cells.

miR-34a and CCNDI are inversely correlated in LSCC tissues. Finally, we examined whether miR-34a and downregulation-mediated CCND1 in clinical LSCC were correlation. We measured the mRNA levels of CCND1 in 23 cases of LSCC specimens and their corresponding normal adjacent tissues by real-time PCR. These cases included 5 cases of clinical stage I, 8 cases of stage II, 8 cases of stage III, and 2 cases of stage IV LSCC. The results indicated that the average expression level of CCND1 was significantly higher in LSCC specimens than that in the corresponding normal adjacent tissues (Fig. 5A; $\mathrm{P}<0.05$ ). We further correlated CCND1 with the miR-34a expression levels in the same LSCC specimens. As shown in Fig. 5B, a significant inverse correlation was observed when CCND1 expression levels were plotted against miR-34a expression levels (2-tailed Spearman's correlation, $\mathrm{r}=-07604 ; \mathrm{P}<0.0001)$.

Cancer is a group of diseases involving uncontrolled growth of abnormal cells, which is caused not only by the change in a series of important proteins but also by a global dysregulation in the miRNA profile (9). miRNAs are conserved small non-coding RNAs that participate in the regulation of a series of fundamental biological processes, such as development, proliferation, apoptosis, differentiation, survival and cell death. In view of the pivotal function of miRNAs, their alteration naturally promotes numerous cell physiological and pathological processes, and are eventually involved in the etiology of many human diseases, such as autoimmune, cardiovascular disease, diabetes, and cancer. In this study, we found that miR-34a was frequently downregulated in LSCC tissues, and its expression level was inversely correlated with lymph node metastasis and advanced clinical stage. Then, restoration of miR-34a suppressed cancer cell proliferation, migration and induced the apoptosis in HEp-2 cells in vitro. Furthermore, we identified CCND1, a putative therapeutic target oncogene (25), as a direct and functional target of miR-34a by binding to the specific 3'-UTR of CCND1. Our study indicated that miR-34a serves as a novel proliferation and metastasis suppressor in LSCC.

As a tumor suppressor microRNA, miR-34a is one of the most characterized miRNAs which has attracted much attention in various cancers. Reduced expression of miR-34a, triggers dysregulation of cell homeostasis, which could be a selective advantage for cancer cells. Indeed, miR-34a is a direct p53 target, and ectopic expression can recapitulate some tumor suppressor biological functions of p53 such as apoptosis, cell cycle arrest, inhibition of cancer stem cells viability and metastasis formation (28). Shen et al showed that miR-34a was downregulated in LSCC using real-time PCR analysis and its levels were negatively correlated with histological differentiation and were positively correlated with survival rate and might be a potential biomarker of progression and prognosis for LSCC. Ectopic expression of miR-34a significantly inhibited cell proliferation by arresting cells at the $\mathrm{G} 0 / \mathrm{G} 1$ phase through targeting survivin in HEp-2 cells (10). However, the miR-34a expression levels in clinical tissues and its definite mechanism in LSCCare not completely understood. In the present study, we demonstrated that miR-34a was downregulated in 
LSCC tissues and the reduced miR-34a expression level was closely correlated to lymphatic node metastasis and advanced TNM stage of LSCC, suggesting that a reduced expression level of miR-34a is associated with LSCC progression. Taken together, these data suggest that miR-34a expression is frequently downregulated in LSCC, and is responsible for the formation and progression of LSCC. However, the exact function of miR-34a in LSCC is not completely elucidated.

Cell proliferation and migration are required for the process of tumorigenesis and progression. miR-34a as an important tumor suppressor miRNA participates in the regulation of multiple steps of the process of cells (13). In the present study, to determine the function of miR-34a in LSCC, we used laryngeal carcinoma cell line HEp-2 as a model, and ectopic expression of miR-34a significantly suppressed HEp-2 cell proliferation and migration (Fig. 2). Our results indicate that miR-34a is a potential therapeutic target for LSCC.

As an important oncogene, more than 100 proteins have been identified to interact with CCND1 in human cancer (29). These proteins are involved in cell cycle control, transcriptional regulation, DNA repair, RNA metabolism, protein folding, cell structure and cell organization. Therefore, the dyregulation of CCND1 affects multiple cellular processes, which could have oncogenic consequences. The overexpression of CCND1 has been shown in $80 \%$ of head and neck squamous cell carcinoma (30). A translocation that juxtaposes CCND1 with the immunoglobulin heavy chain locus, CCND1 copy number amplification, mutations in the 3'-UTR cause stabilization of the CCND1 mRNA, and as a consequence causes oncogenic activation of mitogenic signaling pathways leading to CCND1 overexpression (25). CCND1 overexpression is associated with poor prognosis and increased metastasis. In this study, we identified CCND1 as a target of miR-34a in LSCC, and overexpression of CCND1 was inversely correlated with miR-34a in LSCC clinical tissues. Meanwhile, we found that knockdown of CCND1 caused inhibition of proliferation and migration in HEp-2 cells, and restoration of CCND1 partially abrogated the miR-34a-mediated effects in HEp-2 cells. These results suggest that miR-34a serves as a tumor suppressor miRNA by regulating CCND1 to suppress the proliferation and metastasis of LSCC.

Taken together, our study demonstrated that miR-34a as an important tumor suppressor miRNA is downregulated in LSCC. Ectopic expression of miR-34a inhibited HEp-2 cell proliferation and migration by directly targeting CCND1. These results suggest that the downregulation of miR-34a in LSCC contributes to LSCC tumorigenesis and progression and that miR-34a may be novel diagnostic biomarker and a potential therapeutic target for LSCC.

\section{Acknowledgements}

This study was supported by grants from the Science and Technology Planning Project of Guangdong Province, China (grant no. 2013B021800088 to J.Y., no. 2014A020212477 to LS.L.), and the Natural Science Foundation of Guangdong Province, China (grant no. 2014A030313057 to J.Y., no. 2015A030313035 to LS.L.), and the Specialized Research Fund for the Doctoral Program of Higher Education (grant no. 20130171120069 to LS.L.).

\section{References}

1. Forastiere AA, Zhang Q, Weber RS, Maor MH, Goepfert H, Pajak TF, Morrison W, Glisson B, Trotti A, Ridge JA, et al: Long-term results of RTOG 91-11: a comparison of three nonsurgical treatment strategies to preserve the larynx in patients with locally advanced larynx cancer. J Clin Oncol 31: 845-852, 2013.

2. Bartel DP: MicroRNAs: Genomics, biogenesis, mechanism, and function. Cell 116: 281-297, 2004.

3. Calin GA and Croce CM: MicroRNA signatures in human cancers. Nat Rev Cancer 6: 857-866, 2006.

4. Kasinski AL and Slack FJ: Epigenetics and genetics. MicroRNAs en route to the clinic: progress in validating and targeting microRNAs for cancer therapy. Nat Rev Cancer 11: 849-864, 2011.

5. Chen CZ: MicroRNAs as oncogenes and tumor suppressors. N Engl J Med 353: 1768-1771, 2005.

6. Li L, Luo J, Wang B, Wang D, Xie X, Yuan L, Guo J, Xi S, Gao J, Lin X, et al: Microrna-124 targets flotillin-1 to regulate proliferation and migration in breast cancer. Mol Cancer 12: 163, 2013.

7. Andorfer CA, Necela BM, Thompson EA and Perez EA: MicroRNA signatures: clinical biomarkers for the diagnosis and treatment of breast cancer. Trends Mol Med 17: 313-319, 2011.

8. Banwait JK and Bastola DR: Contribution of bioinformatics prediction in microRNA-based cancer therapeutics. Adv Drug Deliv Rev 81: 94-103, 2015.

9. Berindan-Neagoe I, Monroig PC, Pasculli B and Calin GA: MicroRNAome genome: a treasure for cancer diagnosis and therapy. CA Cancer J Clin 64: 311-336, 2014.

10. Shen Z, Zhan G, Ye D, Ren Y, Cheng L, Wu Z and Guo J: MicroRNA-34a affects the occurrence of laryngeal squamous cell carcinoma by targeting the antiapoptotic gene survivin. Med Oncol 29: 2473-2480, 2012

11. Kumar B, Yadav A, Lang J, Teknos TN and Kumar P: Dysregulation of microRNA-34a expression in head and neck squamous cell carcinoma promotes tumor growth and tumor angiogenesis. PLoS One 7: e37601, 2012.

12. Agostini M and Knight RA: miR-34: From bench to bedside. Oncotarget 5: 872-881, 2014.

13. Hermeking H: The miR-34 family in cancer and apoptosis. Cell Death Differ 17: 193-199, 2010.

14. Tazawa $H$, Tsuchiya $N$, Izumiya $M$ and Nakagama $H$ : Tumor-suppressive miR-34a induces senescence-like growth arrest through modulation of the E2F pathway in human colon cancer cells. Proc Natl Acad Sci USA 104: 15472-15477, 2007.

15. Winton DJ: miR-34a sets the 'sweet spot' for notch in colorectal cancer stem cells. Cell Stem Cell 12: 499-501, 2013.

16. Liu C, Kelnar K, Liu B, Chen X, Calhoun-Davis T, Li H, Patrawala L, Yan H, Jeter C, Honorio S, et al: The microRNA miR-34a inhibits prostate cancer stem cells and metastasis by directly repressing CD44. Nat Med 17: 211-215, 2011.

17. Bandi $\mathrm{N}$ and Vassella $\mathrm{E}$ : $\mathrm{miR}-34 \mathrm{a}$ and $\mathrm{miR}-15 \mathrm{a} / 16$ are co-regulated in non-small cell lung cancer and control cell cycle progression in a synergistic and $\mathrm{Rb}$-dependent manner. Mol Cancer 10: 55, 2011.

18. Dotto GP and Karine L: miR-34a/SIRT6 in squamous differentiation and cancer. Cell Cycle 13: 1055-1056, 2014.

19. Cole KA, Attiyeh EF, Mosse YP, Laquaglia MJ, Diskin SJ, Brodeur GM and Maris JM: A functional screen identifies miR-34a as a candidate neuroblastoma tumor suppressor gene. Mol Cancer Res 6: 735-742, 2008.

20. Li L, Yuan L, Luo J, Gao J, Guo J and Xie X: MiR-34a inhibits proliferation and migration of breast cancer through downregulation of Bcl-2 and SIRT1. Clin Exp Med 13: 109-117, 2013.

21. Li L, Xie X, Luo J, Liu M, Xi S, Guo J, Kong Y, Wu M, Gao J, Xie Z, et al: Targeted expression of miR-34a using the T-VISA system suppresses breast cancer cell growth and invasion. Mol Ther 20: 2326-2334, 2012.

22. Liu X, Lv XB, Wang XP, Sang Y, Xu S, Hu K, Wu M, Liang Y, Liu P, Tang J, et al: MiR-138 suppressed nasopharyngeal carcinoma growth and tumorigenesis by targeting the CCND1 oncogene. Cell Cycle 11: 2495-2506, 2012.

23. Malumbres $\mathrm{M}$ and Barbacid M: Cell cycle, CDKs and cancer: a changing paradigm. Nat Rev Cancer 9: 153-166, 2009.

24. Chen HZ, Tsai SY and Leone G: Emerging roles of E2Fs in cancer: an exit from cell cycle control. Nat Rev Cancer 9: 785-797, 2009. 
25. Musgrove EA, Caldon CE, Barraclough J, Stone A and Sutherland RL: Cyclin D as a therapeutic target in cancer. Nat Rev Cancer 11: 558-572, 2011.

26. Pignataro L, Pruneri G, Carboni N, Capaccio P, Cesana BM, Neri A and Buffa R: Clinical relevance of cyclin D1 protein overexpression in laryngeal squamous cell carcinoma. J Clin Oncol 16: 3069-3077, 1998.

27. Sun F, Fu H, Liu Q, Tie Y, Zhu J, Xing R, Sun Z and Zheng X: Downregulation of CCND1 and CDK6 by miR-34a induces cell cycle arrest. FEBS Lett 582: 1564-1568, 2008.

28. Ahn YH, Gibbons DL, Chakravarti D, Creighton CJ, Rizvi ZH, Adams HP, Pertsemlidis A, Gregory PA, Wright JA, Goodall GJ, et al: ZEB1 drives prometastatic actin cytoskeletal remodeling by downregulating miR-34a expression. J Clin Invest 122: 3170-3183, 2012.
29. Jirawatnotai S, Hu Y, Michowski W, Elias JE, Becks L, Bienvenu F, Zagozdzon A, Goswami T, Wang YE, Clark AB, et al: A function for cyclin D1 in DNA repair uncovered by protein interactome analyses in human cancers. Nature 474 : 230-234, 2011.

30. Leemans CR, Braakhuis BJ and Brakenhoff RH: The molecular biology of head and neck cancer. Nat Rev Cancer 11: 9-22, 2011.

31. Gao J, Li L, Wu M, Liu M, Xie X, Guo J, Tang H and Xie X: MiR-26a inhibits proliferation and migration of breast cancer through repression of MCL-1. PLoS One 8: e65138, 2013. 